\title{
Analyzing Laser-Welded NiTi-NiTi-Joints for Actuator Applications Using Design of Experiments
}

\author{
Dennis Otibar ${ }^{1}$, Christian Rathmann ${ }^{1}$, Konstantin Lygin ${ }^{1}$, Dieter Kreimeier ${ }^{1}$ and Peter Szymansky ${ }^{2}$ \\ 1. Faculty of Mechanical Engineering, Ruhr-University Bochum, Bochum 44801, Germany \\ 2. PSL Technik GmbH, Oberhausen 46149, Germany
}

Received: September 25, 2014 / Accepted: October 20, 2014 / Published: February 25, 2015.

\begin{abstract}
One promising joining method for NiTi-SMA (shape memory alloy)-components is laser welding. This joining technology bears huge potential regarding process automation and mechanical properties as well as durability, especially within the field of smalland medium-sized actuators. However, there is still need for research due to unsolved issues influencing the microstructure and thus effecting mechanical properties as well as SMA-characteristics of these joints. Therefore, the purpose of this paper is the evaluation of quality parameters of NiTi-NiTi-wire-joints. For this purpose, design of experiments with a fractional factorial design is used for the investigation, because of its high potential to decrease experimental effort. This paper provides a basis for future research in the field of SMA-actuators and joining.
\end{abstract}

Key words: SMA, shape memory actuators, NiTi-NiTi-joints, laser welding, joining, design of experiments.

\section{Introduction}

NiTi-SMA (shape memory alloys)-wires are currently used in a wide variety of actuator applications [1]. In many of these applications, proper joining methods are gaining increasing importance due to production process suitability, fatigue strength as well as product requirements like miniaturization or weight reduction. In previous works, it is already shown that laser welding is a suitable joining method for NiTi-SMA [2-5]. These investigations are mainly focused on metallographic examinations as well as on mechanical and functional properties of welded NiTi-NiTi-joints. Thus, no information about NiTi-NiTi-joints for actuator applications is available. Therefore, the purpose of this paper is the evaluation of factors for the laser welding process of NiTi-NiTi-joints by using design of experiments. The paper is organized as follows: Section 2 gives an overview on joining

\footnotetext{
Corresponding author: Christian Rathmann, M.Sc., research field: mechanical engineering. E-mail: Rathmann@lps.ruhr-uni-bochum.de.
}

technologies for SMA; Section 3 focuses on laser welding; Section 4 discusses the experimental design; Section 5 presents the results; finally, Section 6 draws a conclusion and provides an outlook.

\section{Joining of NiTi-SMAs}

The joining of NiTi-SMAs is usually done by adhesive bonding or form locked connections, which include, e.g., crimping, screwing or adhesive bonding. Fig. 1 shows an overview of different joining technologies and their combinations. Most of these joining technologies are only suitable for a limited range of technical applications and increase complexity. Besides that, requirements regarding safety and long-term durability of NiTi-SMA-components are often insufficiently fulfilled. Due to miniaturization of SMA-components in many technical areas, a promising joining method for SMA-components, from a design point of view, is substance-to-substance bonds.

\section{Laser Welding for Joining NiTi-SMA}

One promising joining method for NiTi-SMA-components is laser welding, regarding 


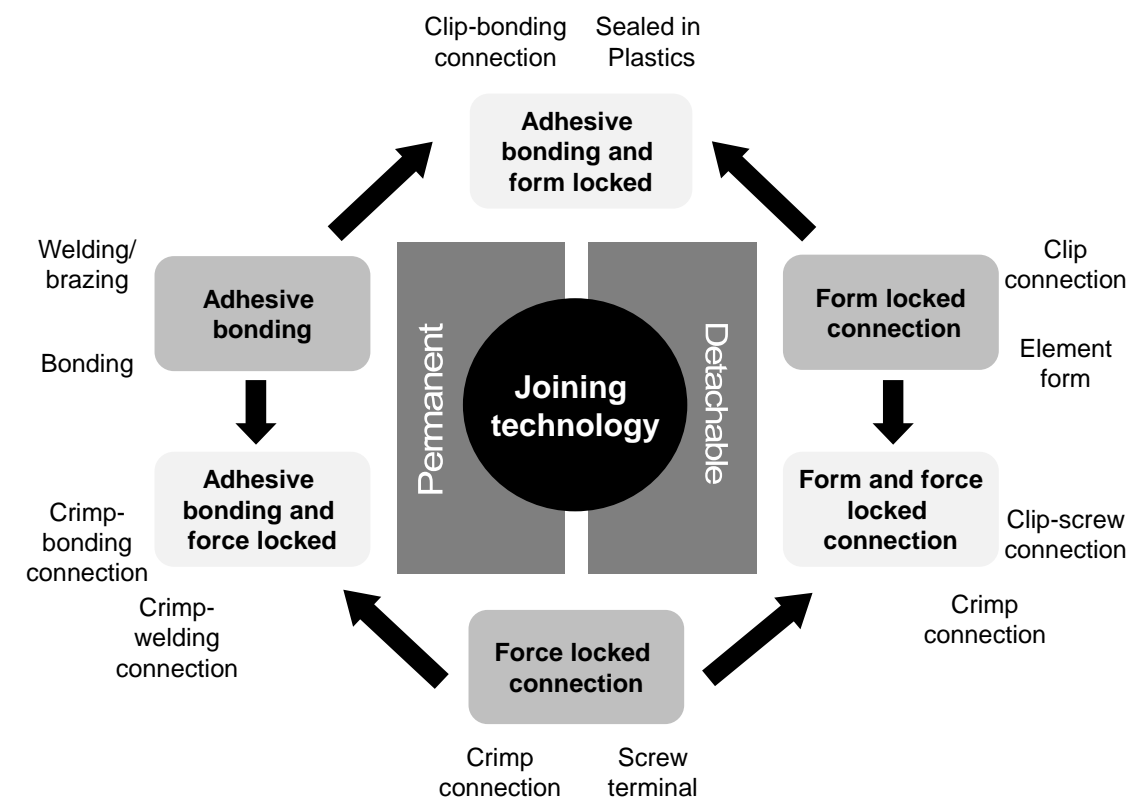

Fig. 1 Overview of possible joining technologies for SMA-actuators [1, 6].

process automation and high mechanical properties as well as durability, especially within the field of smalland medium-sized actuators. In consequence, this technology can lead the way to cut costs significantly and achieve high reliability of the joint. Previous researches on welded NiTi-NiTi-joints focus primarily on medical and micro technology applications $[4,7,8]$ and do not consider dimensions suitable for actuator applications. In addition, a review of existing research shows that, besides investigation regarding automation, embrittlement is still an insufficiently solved issue, influencing the microstructure and thus effecting mechanical properties as well as SMA-characteristics.

The manufacture of the NiTi-NiTi-joints is made by PSL Technik GmbH in Oberhausen, Germany. For this process, a specially designed solid-state laser system with an average power of $150 \mathrm{~W}$ based on Nd:YAG laser technology is developed and used. A laser beam is used to melt the surface of a specimen (Fig. 2). Major advantages of the Nd:YAG laser technology is high precision welding especially for thin materials [9].

Due to technical modifications, the adjustment range of the spot size and pulse duration are optimized for minimization of the heat affected zone and the melting zone. Regarding the sensitivity to temperature of SMA-materials, these parameters are of great importance. A minimization of the influences on mechanical and functional SMA-properties due to laser shots should be achieved.

\section{Material and Methods}

\subsection{Material}

Due to their practical importance for actuator applications, only SMA-wires are considered. A Nd:YAG laser is used for laser welding. For all experiments, Memry NiTi-alloy N or B with $0.4 \mathrm{~mm}$ diameter is used. To determine strain and tensile

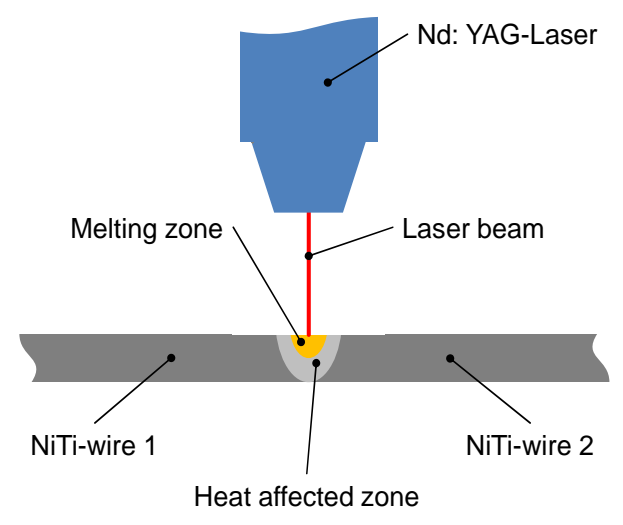

Fig. 2 Laser welding of NiTi-wires. 
strength, a tensile testing machine type T300 which meets the standard DIN EN ISO 7500-1:2014-05 is used.

\subsection{Experiment Design}

DoE (design of experiments) is used to investigate and evaluate the quality of laser welded NiTi-NiTi-joints regarding the identification of significant factors and interactions between factors and response. DoE offers a systematical approach with various tools based on statistics, in which several factors are simultaneously changed.

Fig. 3 illustrates an example of a $2^{3-1}$ fractional factorial design, which is best for factor screening. This design allows drastically reducing sample size and is best for identifying important factors. Nevertheless, it can imply significant risk since factors are mixed [10]. A broad variety of designs exists like full factorial design, Taguchi design for stable process or designs, and central composite design for non-linear interrelations.

\subsection{Experimental Procedure}

Ref. [1] is analyzed to gather initial situation about welded joining of NiTi-SMA and is discussed with industry experts.

After this, response and factors are selected. For the response, the tensile strength and strain represents important responses, which are analyzed. Therefore, the experiments focus primarily on mechanical properties of welded NiTi-SMA-joining and the influence on NiTi-SMA-characteristics. Based on this, a fractional factorial design is used.

Selection of investigated factors is based on a factor-response-matrix. In this matrix, every factor from a previous given brainstorming is listed and criteria are used for evaluation. Criteria to evaluate the importance for welding process are:

- influence on response (tensile strength and strain);

- modification effort;

- reproducibility;

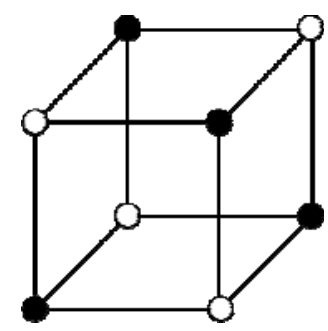

Fig. $32^{3-1}$ fractional factorial design.

- cost of changing.

Based on this, the investigated factors are alloy composition, duration of heat treatment, duration of pulse, laser power, type of separating process, temperature of heat treatment, number of welding spots, type of chemical surface pretreatment and spot diameter. In Table 1, all factors and the investigated levels are listed.

A $2^{8-2}$ fractional factorial design with resolution $\mathrm{V}$ is used for the investigation. The major advantage of this experimental design is that all factors and two-factor interactions are determinable, with reduced sample size. Due to this, the investigation covers 64 samples.

In preparation of the experiments, a trail run is performed to ensure the feasibility of experiments. It becomes obvious that bending of the NiTi-wires-endings is an important issue for the welding process as well as surface roughness which has an influence on the response. Besides that, the reproducibility of a manual welding process proved to be sufficient.

\section{Results}

The evaluation of the experiments is supported by the statistic software JMP 10. Prior to analyzing the

Table 1 Factors and levels of the welding process.

\begin{tabular}{llll}
\hline Factors & Unit & \multicolumn{2}{c}{ Level } \\
\hline Alloy composition & - & $\mathrm{N}$ & $\mathrm{B}$ \\
Duration of heat treatment & $\min$ & 5 & 20 \\
Duration of pulse & $\mathrm{m} \cdot \mathrm{s}^{-1}$ & 1.75 & 1.85 \\
Laser power & $\mathrm{J}$ & 190 & 200 \\
Separating process & - & $\mathrm{Cutting}$ & Filling \\
Temperature of heat treatment & ${ }^{\circ} \mathrm{C}$ & 300 & 500 \\
Number of welding spots & - & 1 & 2 \\
Type of chemical surface cleanliness & - & Grinding & Ethanol \\
\hline
\end{tabular}




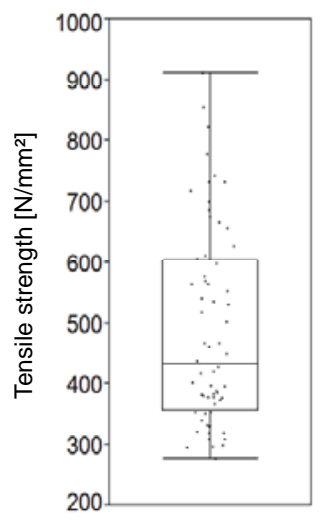

(a)

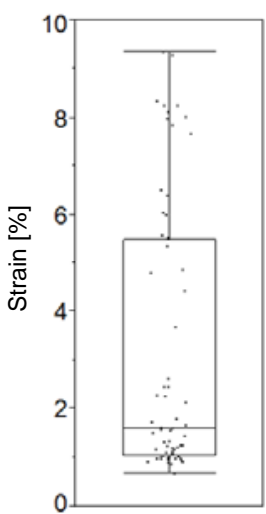

(b)
Fig. 4 Box plot of (a) tensile strength and (b) strain.

experiments, the gathered data are checked for plausibility. The knowledge-based and statistical-based control, using box-plot, shows one abnormality, which is excluded. Abnormalities are response values outside average plus or minus two and a half times the standard deviation (Fig. 4).

\subsection{Response: Tensile Strength}

The tensile strength of laser welded NiTi-NiTi-wire-joints varies from $276 \mathrm{~N} \cdot \mathrm{mm}^{-2}$ up to $854 \mathrm{~N} \cdot \mathrm{mm}^{-2}$. The lower quartile is at $354 \mathrm{~N} \cdot \mathrm{mm}^{-2}$, and the upper quartile is at $598 \mathrm{~N} \cdot \mathrm{mm}^{-2}$ [11]. Compared with unwelded NiTi-wires with 800-1,000 N·mm ${ }^{-2}$ tensile strength, the tensile strength of the welded NiTi-wires is reduced by almost half. Noteworthy is that there are results, which have almost the tensile strength of the unwelded NiTi-wires. Possible reasons for this are analyzed in the following parts.

By using effect-screening with a level of significance of 95\%, the following factors and factor interactions of the second order are considered to be significant for the response tensile strength:

- number of welding spots;

- alloy composition;

- duration of heat treatment and duration of pulse;

- duration of pulse and separating process;

- alloy composition and type of chemical surface cleanliness;

- duration of heat treatment and type of chemical surface cleanliness.
Interestingly enough, no factors concerning the welding process are significant, although NiTi-wires are very energy-sensitive. One reason for this may be the high precision and very low energy input of this welding process. Probably other welding processes have a greater influence on the response. Furthermore, one must consider that tensile strength of welded NiT-NiTi-joints for other diameter can be influenced by other factors.

\subsection{Response: Strain}

The strain of laser welded NiTi-NiTi-wire-joints varies from $0.68 \%$ up to $9.33 \%$. The lower quartile is at 1.02, and the upper quartile is at 5.36. Regarding the results for the response, one must consider that the NiTi-wires are in the martensite phase, and therefore, the strain cannot be compared with the typical reversible strain of this material.

By using effect-screening with a level of significance of $95 \%$, the following factors and factor interactions of the second order are considered to be significant for the response strain:

- number of welding spots;

- temperature of heat treatment;

- duration of heat treatment;

- temperature of heat treatment and number of welding spots;

- duration of heat treatment and duration of pulse;

- temperature of heat treatment and duration of heat treatment;

- duration of pulse and type of chemical surface cleanliness;

- duration of heat treatment and number of welding spots;

- duration of heat treatment and separating process. Similar to results of tensile strength, the number of welding points has the largest influence on the response. After this, factors concerning heat treatment are relevant. This makes sense since heat treatment affects the crystal structure of the material, and thus reduces dislocations and hardening. Fig. 5 illustrates the interactions between temperature and duration of heat 


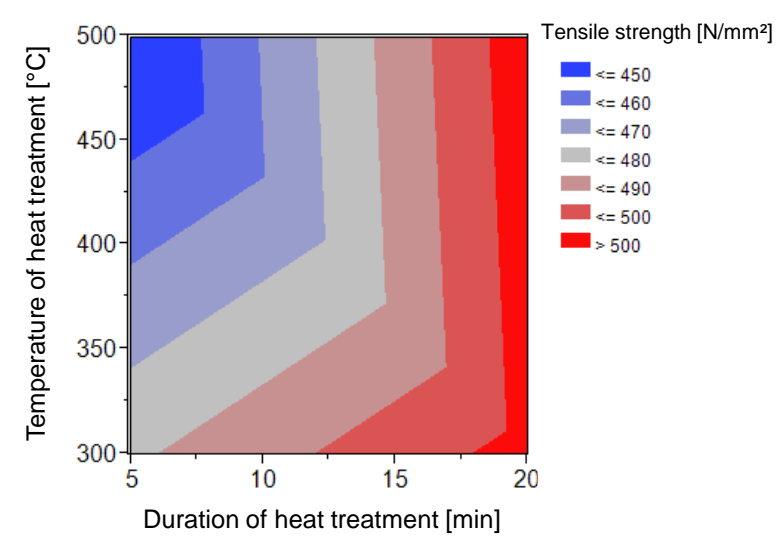

(a)

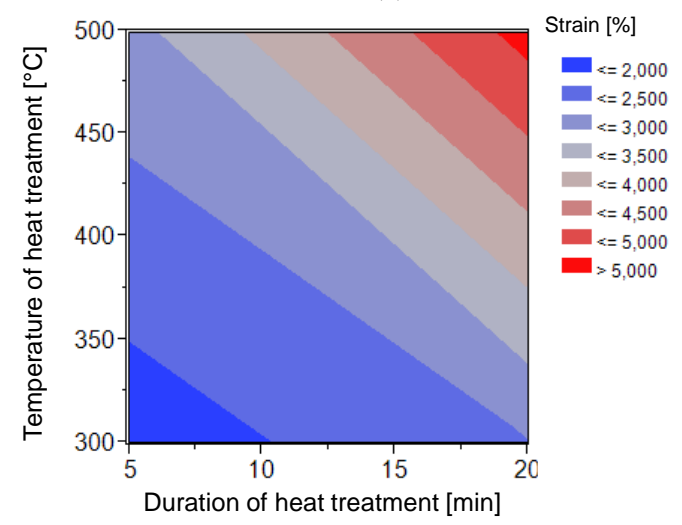

(b)

Fig. 5 Conture plot of (a) tensile strength and (b) strain.

treatment. Higher temperature hand in hand with longer heat treatment increases the possible strain. Thus, it is interesting to investigate from what temperature and duration the mechanical properties decreases again or if this is already the optimum for welded NiTi-NiTi-joints. In contrast to the response, tensile strength two-factor interactions are of minor importance.

\subsection{Response: Tensile Strength and Strain}

The relationship illustrated between tensile strength and strain in Fig. 6 can be proved mathematically by a non-linear regression analysis. The result is a polynomial of third degree with a coefficient of determination of almost $80 \%$, which verifies the strong relationship.

The optima for both responses can be found in Table 2. This optima has a tensile strength of $855 \mathrm{~N} \cdot \mathrm{mm}^{-2}$ and a strain of $9.34 \%$.

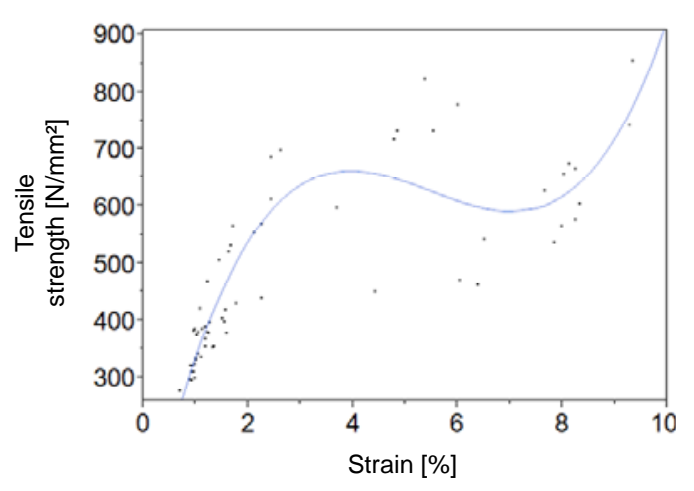

Fig. 6 Stress-strain diagram with tensile strengths of all specimens.

Table 2 Optima for maximum tensile strength and strain.

\begin{tabular}{lll}
\hline Factors & Unit & Level \\
\hline Alloy composition & - & $\mathrm{N}$ \\
Duration of heat treatment & $\mathrm{min}$ & 20 \\
Duration of pulse & $\mathrm{m} \cdot \mathrm{s}^{-1}$ & 1.85 \\
Laser power & $\mathrm{J}$ & 200 \\
Separating process & - & Filling \\
Temperature of heat treatment & ${ }^{\circ} \mathrm{C}$ & 500 \\
Number of welding spots & - & 1 \\
Type of chemical surface cleanliness & - & Ethanol \\
Strain & $\%$ & 9.34 \\
Tensile strength & $\mathrm{N} \cdot \mathrm{mm}^{-2}$ & 854.98 \\
\hline
\end{tabular}

This result indicates that the tensile strength value depends on the material composition whereas the strain can be influenced significantly by the heat treatment. As a conclusion for NiTi-NiTi welding procedures, it can be said that the material composition has to be selected first to obtain optimal mechanical properties. Thereafter, the selection of parameter for heat treatment and thus setting of functional properties has to follow.

\section{Conclusions and Outlook}

Even though welding has a negative influence on mechanical properties of NiTi-SMA-joining, it is possible to use signification factors (number of welding spots, alloy composition, temperature of heat treatment and duration of heat treatment) to improve mechanical properties of the NiTi-SMA-joining. With the identified factors, it is possible to adjust the properties to requirements in certain range. This makes it possible to expand the range of application for SMA. 
This paper focuses on laser-welded NiTi-NiTi-joints, which can be seen as a starting point for further research. Further researches should cover dissimilar material joining. In this context, for the integration of NiTi-components in a mechanical system or the casing, a welded joint of Al-NiTi is an important requirement for large scale production of shape memory actuators. Additionally, investigating electrical contacting of shape memory actuators namely via combination of $\mathrm{Cu}-\mathrm{NiTi}$ can increase the number of possible applications.

\section{Acknowledgments}

The authors acknowledge funding of the hyProFGA project by the NRW (North Rhine-Westphalia).Bank through

Ziel2.NRW-programm

Automotive+Produktion.NRW.

\section{References}

[1] Langbein, S., and Czechowicz, A. 2013. Design Practice Shape Memory Technology_Potentials_Calculation-Examples. Munich: Carl Hanser Press.

[2] Gugel, H. 2010. "Laser Welding of Similar Type and Unlike Kind Combinations of Material with
Nickel-Titanium Shape Memory Alloys.” Dissertation, Bochum, Germany.

[3] Schürmann, A. 2005. "Joining and Processing of Material Composites with Nickel-Titanium Shape Memory Alloys.” Dissertation, Bochum, Germany.

[4] Haas, T., and Schüssler, A. 1995. "Laser Welding of NiTi-Alloys with Memory Effect.” Laser Magazine 1 (95): 46-50.

[5] Falvo, A., Furgiuele, F. M., and Maletta, C. 2005. "Laser Welding of a NiTi Alloy: Mechanical and Shape Memory Behavior.” Materials Science and Engineering: A 412 (1-2): 235-40.

[6] Lygin, K., Langbein, S., and Sadek, T. 2010. "Development and Test of Force-Locked Connecting Elements for Shape Memory Alloy Wires.” Presented at the SMST Conference, Pacific Grove, USA.

[7] Gugel, H., Schuermann, A., and Theisen, W. 2008. "Laser Welding of NiTi Wires." Materials Science and Engineering: A 481-482: 668-71.

[8] Song, Y. G., Li, W. S., Li, L., and Zheng, Y. F. 2008. "The Influence of Laser Welding Parameters on the Microstructure and Mechanical Property of the As-Jointed NiTi Alloy Wires.” Materials Letters 62: 2325-8.

[9] McGeough, J. A. 1988. Advanced Methods of Machining. London: Chapman and Hall.

[10] Kleppmann, W. 2013. Design of Experiments-Optimizing Products and Processes. Munich: Carl Hanser Press.

[11] Hartmut, J. 2010. Unconventional Actuators-An Introduction. München: Oldenbourg. 